\title{
Structured catalysts for partial oxidations
}

\author{
Victor J.M. de Campos, Rosa M. Quinta-Ferreira* \\ Department of Chemical Engineering, University of Coimbra, Portugal Pólo II, Pinhal de Marrocos, 3030 Coimbra, Portugal
}

\begin{abstract}
The objective of the present work is centred on the analysis of the behaviour of fixed-bed reactors packed with structured catalysts when a partial oxidation takes place. The fluid flow within an element, formed by the intersection of two adjacent corrugated plates, was mathematically modelled and numerical simulations were performed in order to study possible optimising of the catalyst structure. A simple model, involving the continuous stirred tank approach, was used to simulate the performance of the catalyst in an industrial reactor. The profiles of temperature, concentration, velocity and pressure along the reactor were obtained through the global mass balance, partial molar balances, conservation of moment, energy balance and mechanical energy balance. These equations were applied to an exothermic reactional system, the selective oxidation of methanol to formaldehyde, in order to evaluate the possibility of application of these catalysts in industry. Various parameters specific to the catalyst and the reactional system were tested in order to achieve a better understanding of the behaviour of these structured packing. The comparison with the heterogeneous model predictions for a random packing and with industrial values pointed out that the choice of parameters is fundamental to the performance of the catalyst. The adjustments to the parameters allows for significant improvements in some of the more troublesome aspects of the reactional system. Lowering the hot-spot, reducing the progress of the secondary reaction and reducing the pressure drop are the main enhancements that these structured catalysts can offer. () 2001 Elsevier Science B.V. All rights reserved.
\end{abstract}

Keywords: Partial oxidations; Fixed-bed reactors; Structured catalysts

\section{Introduction}

The vast majority of the fixed-bed reactors used in industry are randomly packed with catalyst particles of different shapes. These systems normally exhibit some problematic aspects in fluid flow, such as, high resistance to heat removal, stagnated zones, high pressure drops. On the other hand, reactors with monolithic embedding either parallel canals or honeycombs, reduce the drop in pressure but lower the capacity of mass and heat transfer. However, the structured supports characterised by a fixed structured form with a non-chaotic fluid flow, also known as static mixers, can be envisaged as a hybrid between those two types

\footnotetext{
* Corresponding author.

E-mail address: eq1rqf@eq.uc.pt (R.M. Quinta-Ferreira).
}

of packing, the random and the monolithic packing. The use of static mixers has had a vast application in the chemical industry, nominally in separation and absorption columns. Its advantages in these areas include the lack of moving parts and its high capacity to homogenise concentrations and temperatures [1]. Recently, arose the possibility of their use in the area of chemical reaction engineering in heterogeneous processes. Structured catalysts provoke divisions and rearrangements in the fluid currents, intensifying the turbulence of the current. By this it is able to raise the heat transfer from the interior to the reactor wall, without the high pressure drops. Each element of the packing is placed into the reactor at a $90^{\circ}$ angle in relation to the previous one in order to promote the fluid mixing. In this work, the KATAPAK catalyst from Sulzer Chemtech was used [2]. The catalyst 


\begin{tabular}{|ll|}
\hline Nomenclature \\
$a$ & half corrugation height \\
$A$ & passage area $\left(\mathrm{m}^{2}\right)$ \\
$c_{p_{i}}$ & heat capacity of the current \\
& $i\left(\mathrm{~J} \mathrm{~mol}^{-1} \mathrm{~K}^{-1}\right)$ \\
$e$ & width of the plate \\
$E$ & friction loss of energy \\
$\mathrm{HT}$ & heterogeneous model for \\
& random packing \\
$-\Delta H$ & heat of reaction $(\mathrm{J} / \mathrm{mol})$ \\
$\mathrm{ncD}$ & number of radial cells \\
$\mathrm{ncL}$ & number of axial cells \\
$p_{i}$ & current $i$ pressure $($ atm $)$ \\
$P$ & total pressure $(\mathrm{atm})$ \\
$R$ & ideal gas constant $\left(\right.$ atm $\left.\mathrm{m}^{3} \mathrm{~mol}^{-1} \mathrm{~K}^{-1}\right)$ \\
$R_{1}$ & reaction rate of the main \\
& reaction (mol/(kg s)) \\
$R_{2}$ & reaction rate of the side \\
& reaction (mol/(kg s)) \\
$T$ & cell volume temperature $(\mathrm{K})$ \\
$T_{i}$ & current $i$ temperature $(\mathrm{K})$ \\
$v_{i}$ & fluid velocity in the current $i\left(\mathrm{~m} \mathrm{~s}^{-1}\right)$ \\
$x_{\mathrm{A} i}$ & molar fraction of the component \\
& A in current $i$ \\
$Y_{\mathrm{M}}$ & methanol molar fraction \\
$z^{*}$ & normalised reactor length \\
Greek & symbols \\
$\lambda$ & corrugation length \\
$\xi$ & friction coefficient \\
$\rho$ & fluid density $\left(\mathrm{kg} / \mathrm{m}^{3}\right)$ \\
$\varphi$ & crossflow angle (rad) \\
Subscripts \\
$\mathrm{F}$ & formaldehyde \\
$\mathrm{M}$ & methanol \\
0 & reactor entrance \\
\hline
\end{tabular}

element is formed by corrugated plates made of metal or ceramics and the corrugations of these plates are characterised by some specific parameters $[3,4]$ : length, $\lambda$, height, $2 a$, and crossflow angle, $\varphi$, as shown in Fig. 1. Because of the fixed structure of the catalyst, it is possible to determine the flow of a fluid in each corrugation [5]. It is also possible to delimit an elemental volume, which will be the base for the balances. This cell element can be easily visualised by looking

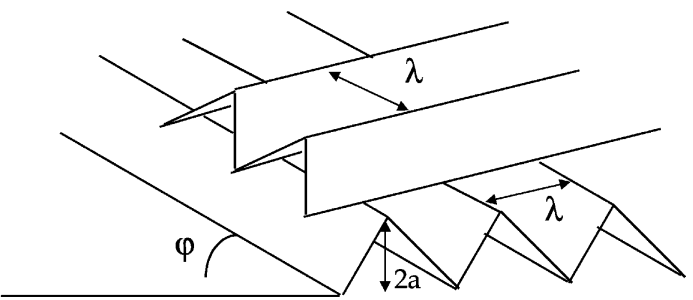

Fig. 1. Two adjacent corrugated sheets.

at two adjacent corrugated plates, Fig. 2. Based on the work of Stoter et al. [6], one can consider this element as a CSTR and the space between two plates as a matrix of tanks, Fig. 3. Each tank has two entrances and two exits and the values of the molar fractions and the temperature of the exit currents were considered to be equal to those occurring inside the cell. Being necessary to calculate the profiles of concentration, temperature, velocity and pressure throughout the reactor, the following balances were required [7]: global mass balance; partial molar balances; conservation of momentum; energy balance; mechanical energy balance.

In this work, we analysed the behaviour of such a system where the selective oxidation of the methanol to formaldehyde occurs according the main reaction for the formaldehyde production and also a side reaction consuming this compound:

$$
\begin{aligned}
& \mathrm{CH}_{3} \mathrm{OH}+\frac{1}{2} \mathrm{O}_{2} \rightarrow \mathrm{CH}_{2} \mathrm{O}+\mathrm{H}_{2} \mathrm{O}, \\
& \mathrm{CH}_{2} \mathrm{O}+\frac{1}{2} \mathrm{O}_{2} \rightarrow \mathrm{CO}+\mathrm{H}_{2} \mathrm{O}
\end{aligned}
$$

The kinetic expressions referred below were used to describe both reactions [8,9]:

$$
R_{1}=k_{1}(T) P^{0.75} \frac{Y_{\mathrm{M}}^{0.75}}{\left(1+Y_{\mathrm{M}}^{0.5}\right)^{0.5}}, \quad R_{2}=k_{2}(T) P_{\mathrm{CH}_{2} \mathrm{O}}
$$

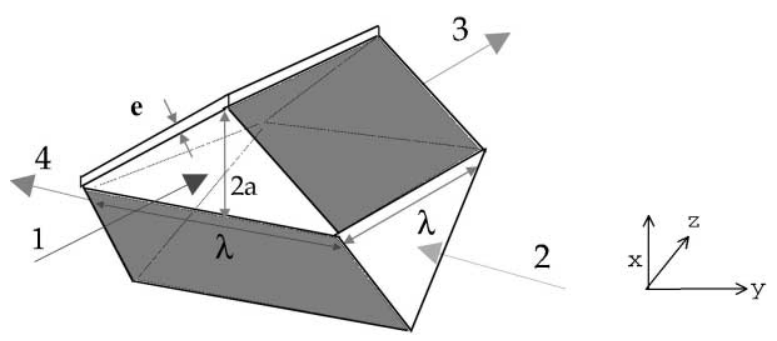

Fig. 2. The cell. 


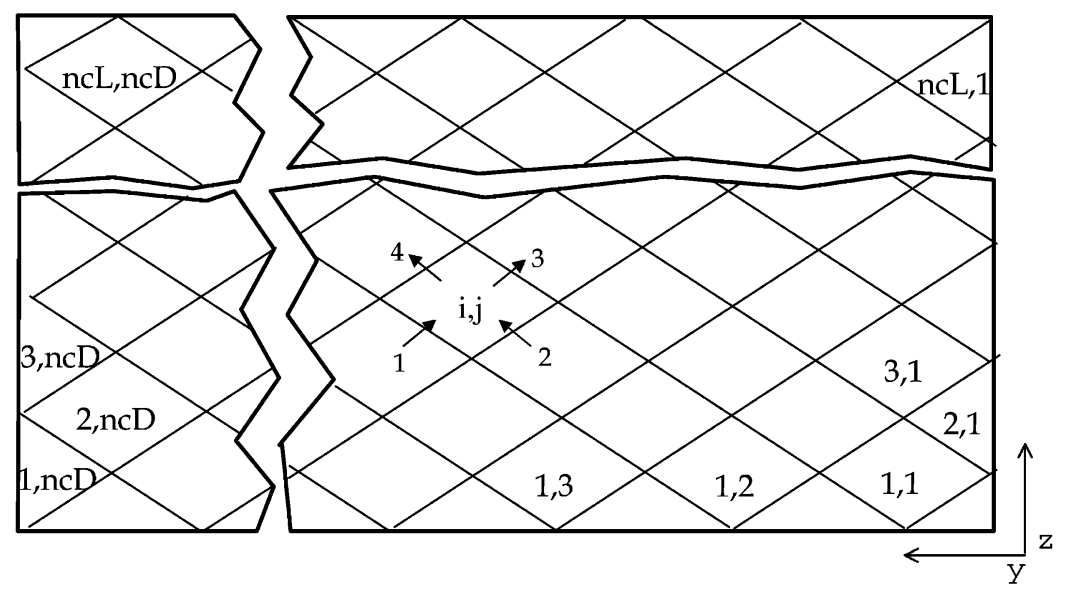

Fig. 3. Matrix system of tanks.

with $k_{1}(T)=k_{0,1} \exp \left(-E_{a, 1} / R T\right), k_{2}(T)=$ $k_{0,2} \exp \left(-E_{a, 2} / R T\right), E_{a, 1}=36,786 \mathrm{~J} / \mathrm{mol}, E_{a, 2}=$ $66,413 \mathrm{~J} / \mathrm{mol}, k_{0,1}=2.92 \times 10^{2} \mathrm{~mol} /(\mathrm{kg} \mathrm{s} \mathrm{atm})^{0.75}$, and $k_{0,2}=3.00 \times 10^{3} \mathrm{~mol} /(\mathrm{kg} \mathrm{s}$ atm $)$.

\section{Mathematical model}

The global mass balance quantifies the conservation of total mass in the system. Since we are considering $\rho$ constant, this balance reduces itself to a velocity equilibrium:

$v_{1}+v_{2}=v_{3}+v_{4}$

The partial balances for each component are written in terms of mole fraction because of the fact that as we considered a CSTR, the fractions (and not the concentration) of each component in the exit current are constant, that is, the molar fraction of the component $A$ is the same as in current 3 and in current 4 (Fig. 3):

$$
\frac{P A}{R T}\left(x_{\mathrm{A} 1} v_{1}+x_{\mathrm{A} 2} v_{2}-x_{\mathrm{A}} v_{3}-x_{\mathrm{A}} v_{4}\right)+R_{\mathrm{A}}^{\prime}=0
$$

$R_{\mathrm{A}}^{\prime}$ is the reaction rate of the component $\mathrm{A}$ referred to the volume of the cell, $V: R_{\mathrm{A}}^{\prime}=R_{\mathrm{A}} \rho_{\mathrm{b}} V$, being $R_{\mathrm{A}}$ the reaction rate referred to the mass of the catalysts $\left(\mathrm{mol} /\left(\mathrm{kg}_{\mathrm{cat}} \mathrm{s}\right)\right)$ and $\rho_{\mathrm{b}}$ the density of the catalyst in the reactor $\left(\mathrm{kg}_{\text {cat }} / \mathrm{m}_{\mathrm{r}}^{3}\right)$.
The conservation of the momentum furnishes two equations: one for the $y$-axis, and another for the $z$-axis, that quantify pressure variation, always present in fixed-bed reactors:

$$
\begin{aligned}
y \text {-axis : } & \frac{1}{2} \xi \rho \cos \varphi\left(-v_{1}^{2}+v_{2}^{2}-v_{3}^{2}+v_{4}^{2}\right) \\
& +\cos \varphi\left(p_{1}-p_{2}-p_{3}+p_{4}\right) \\
= & \rho \cos \varphi\left(-v_{1}^{2}+v_{2}^{2}+v_{3}^{2}-v_{4}^{2}\right) \\
z \text {-axis : } \quad & -\rho \sin \varphi\left(-v_{1}^{2}-v_{2}^{2}-v_{3}^{2}-v_{4}^{2}\right) \\
& +\cos \varphi\left(p_{1}+p_{2}-p_{3}-p_{4}\right) \\
& =\rho \cos \varphi\left(-v_{1}^{2}-v_{2}^{2}+v_{3}^{2}+v_{4}^{2}\right)
\end{aligned}
$$

The energy balance determines the temperature in the element volume:

$$
\begin{aligned}
\rho A & \left(v_{1} c_{p_{1}} T_{1}+v_{2} c_{p_{2}} T_{2}-v_{3} c_{p_{3}} T_{3}-v_{4} c_{p_{4}} T_{4}\right) \\
& +\frac{1}{2}\left(\rho_{1} v_{1}^{3}+\rho_{2} v_{2}^{3}-\rho_{3} v_{3}^{3}-\rho_{4} v_{4}^{3}\right) \\
& +\sum\left(-\Delta H_{j}\right) R_{j}=0
\end{aligned}
$$

The mechanical energy balance was included because of the important role that friction plays in this catalyst [10]:

$$
\begin{aligned}
E_{v}= & \frac{1}{2} \rho A\left(v_{1}^{3}+v_{2}^{3}-v_{3}^{3}-v_{4}^{3}\right) \\
& +A\left(p_{1} v_{1}+p_{2} v_{2}-p_{3} v_{3}-p_{4} v_{4}\right)
\end{aligned}
$$


The values of the entrance variables in each cell, 1 and 2, are known. Therefore, the unknown variables are $x_{\mathrm{A}}$ (A stands for methanol and formaldehyde), $T$, $v_{3}, v_{4}, P_{3}$, and $P_{4}$. This system of non-linear algebraic equations was solved by using a NAG code, which makes use of the modified hybrid Powell method.

\section{Computer results}

The cell model we used allowed the determination of axial and radial profiles of the methanol and formaldehyde concentration, temperature, pressure and velocity. The longitudinal profiles were taken from the central cells and the radial profiles were taken from the hot-spot, where the highest radial gradients occur. Figs. 4-8 represent the behaviour of the system for different values of the amplitude of the corrugation, $\lambda$. This parameter has an important influence on the volume of the cell and therefore on the number of longitudinal and transversal cells. The behaviour of the temperature (Fig. 4) reflects this influence. These results can also be compared with the axial profiles we obtained in a previous study with a heterogeneous model, HT, for a random packing with two catalytic zones [11] and also with some data obtained in an industrial reactor packed with the conventional hollow cylinders. It can be observed that it is possible to select $\lambda$ values that will be favourable to the process. The axial temperature oscillates for $\lambda=7 \mathrm{~mm}$ (Fig. 4a), due to the low number of transversal cells which is only one and a half. The "central" cell being analysed is the one that is closest to the right wall. This cell is alternately a whole interior cell and a half cell adjacent to the wall. The cells adjacent to the wall suffer a higher heat transfer, having therefore lower temperatures than the interior cells, thus the oscillation. The temperature difference for $\lambda=3$ and $5 \mathrm{~mm}$ reflects the fact that lower values of $\lambda$ originate more corrugations, which lead to a higher quantity of catalyst per volume of reactor, with a consequent rise in the reaction yield (raising the temperature). In Fig. 4b, where the radial temperature profiles for the hot-spots are shown, one can also observe that the higher temperature gradients are for lower values of $\lambda$.

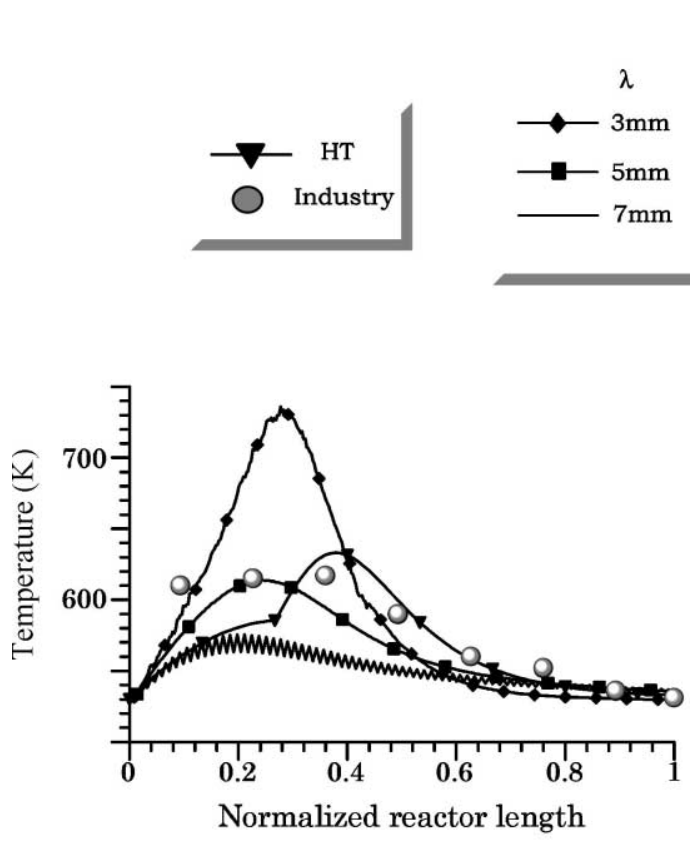

(a)

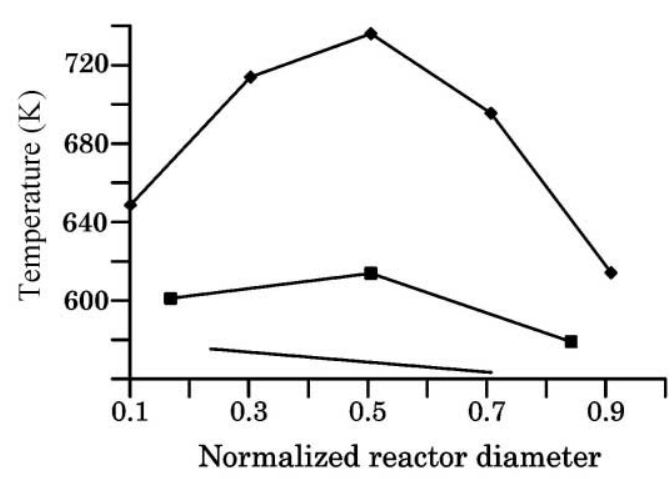

(b)

Fig. 4. Temperature profiles for different $\lambda$ values: (a) longitudinal profiles in the reactor centre; (b) radial profiles in the hot-spots $\left(z^{*}\right)$. 


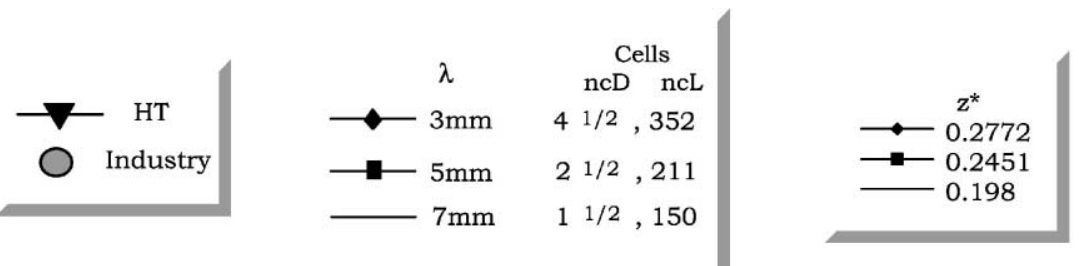

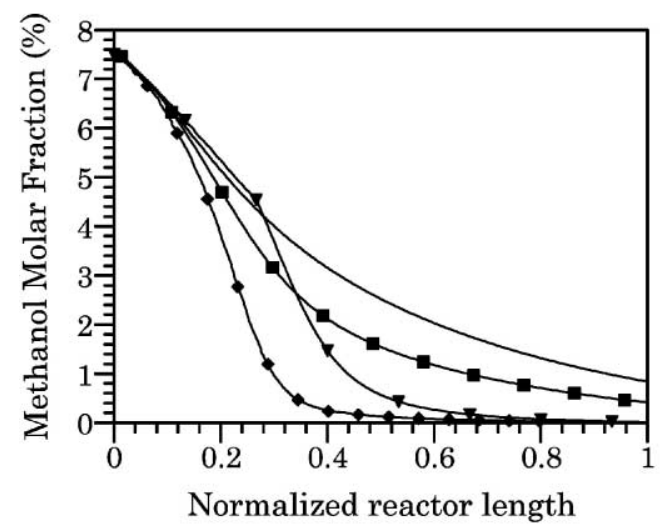

(a)

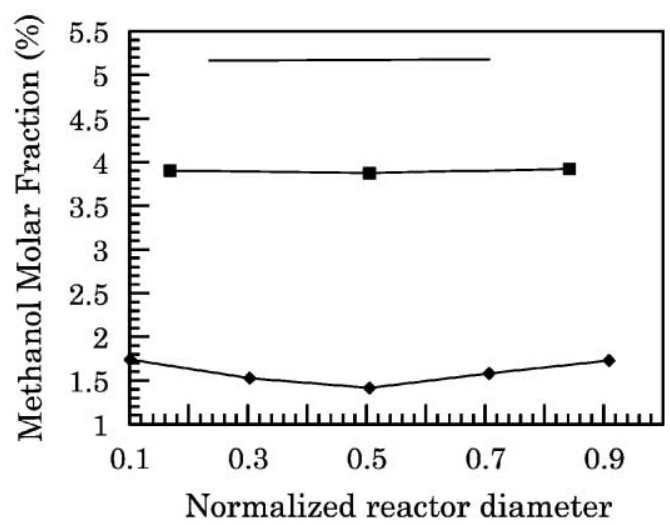

(b)

Fig. 5. Methanol molar fraction profiles for different $\lambda$ values: (a) longitudinal profiles in the reactor centre; (b) radial profiles in the hot-spots $\left(z^{*}\right)$.

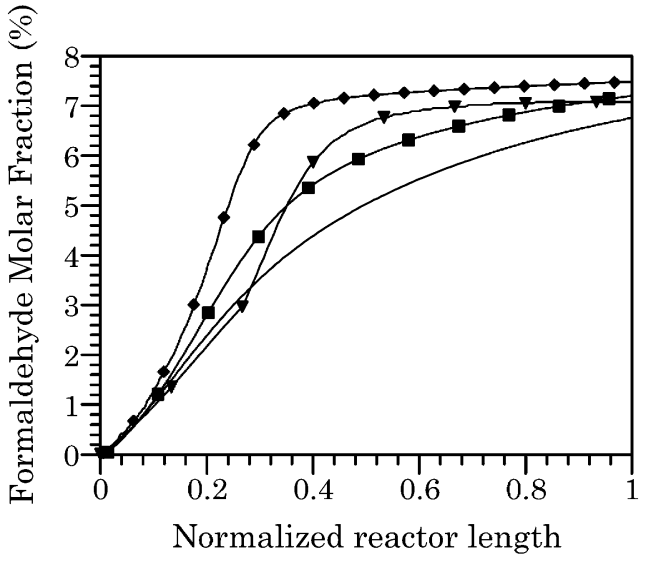

(a)

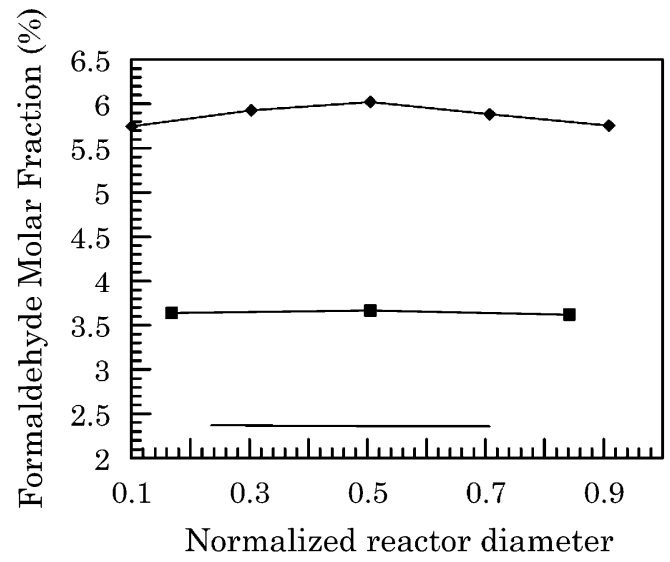

(b)

Fig. 6. Formaldehyde molar fraction profiles for different $\lambda$ values: (a) longitudinal profiles in the reactor centre; (b) radial profiles in the hot-spots $\left(z^{*}\right)$. 


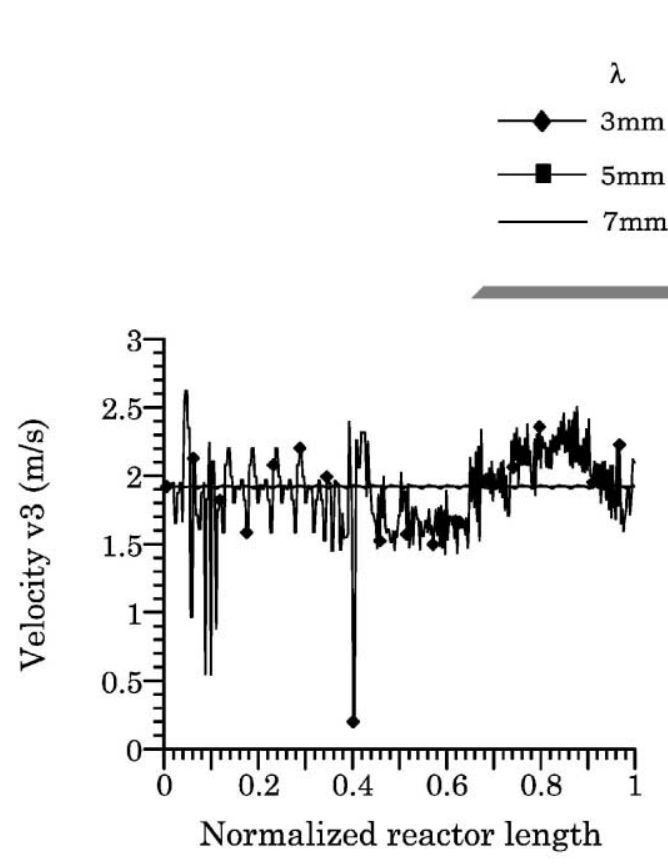

(a)
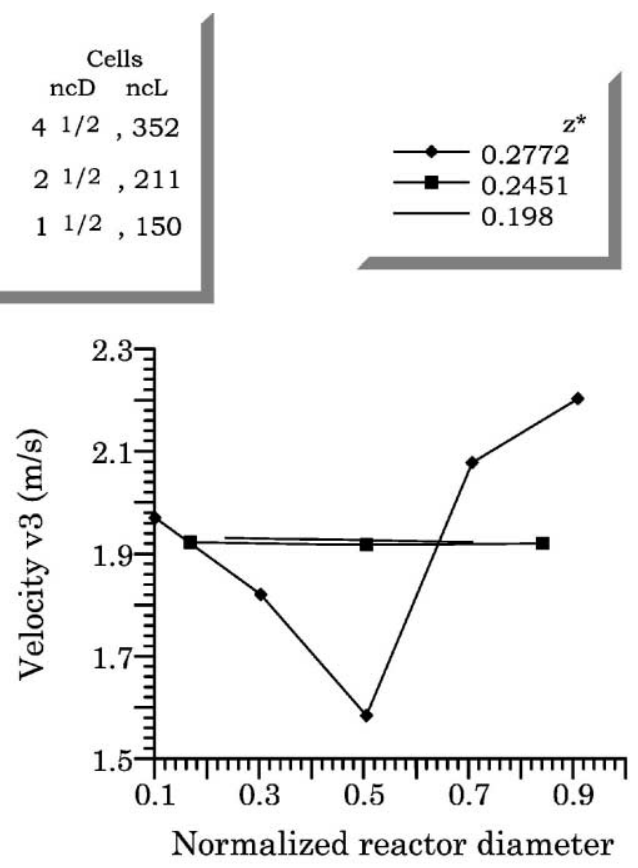

(b)

Fig. 7. Velocity profiles, $v_{3}$, for different $\lambda$ values: (a) longitudinal profiles in the reactor centre; (b) radial profiles in the hot-spots $\left(z^{*}\right)$.

The results on the reactor temperature have their correspondence in the molar fractions of methanol and formaldehyde, Figs. 5a and 6a for longitudinal profiles and Figs. 5b and $6 \mathrm{~b}$ for the radial profiles. Higher temperatures imply greater conversions of methanol to formaldehyde, being negligible the radial gradients of the molar fractions of these compounds. Concerning the fluid velocities, Fig. 7 shows that when $\lambda=3 \mathrm{~mm}$ there exists a large oscillation throughout the reactor. For the other values of $\lambda$, the velocity remains relatively constant. Fig. 8 shows the variation of the pressure, $P_{3}$, where it can be observed that the

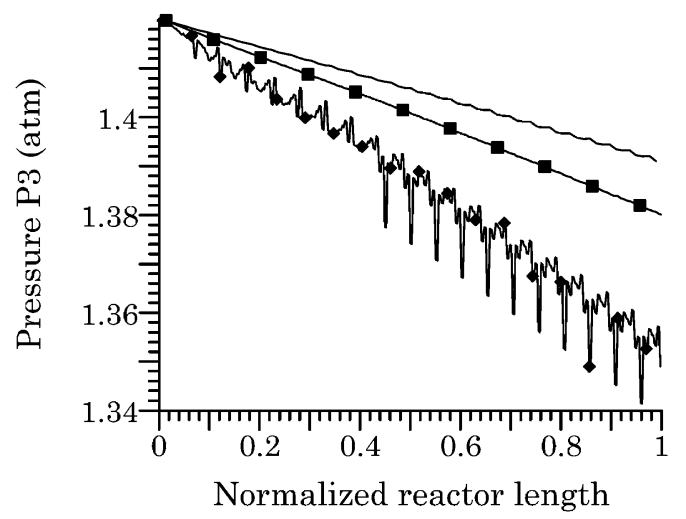

(a)

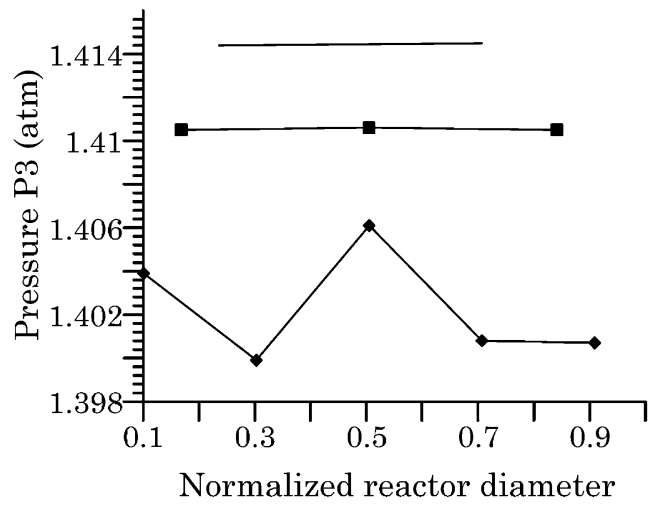

(b)

Fig. 8. Pressure profiles, $P_{3}$, for different $\lambda$ values: (a) longitudinal profiles in the reactor centre; (b) radial profiles in the hot-spots $\left(z^{*}\right)$. 


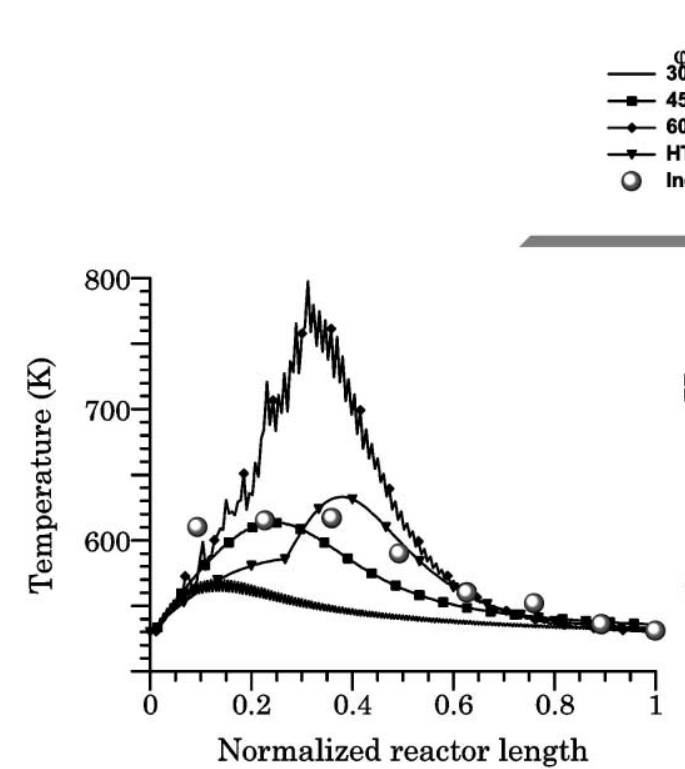

(a)

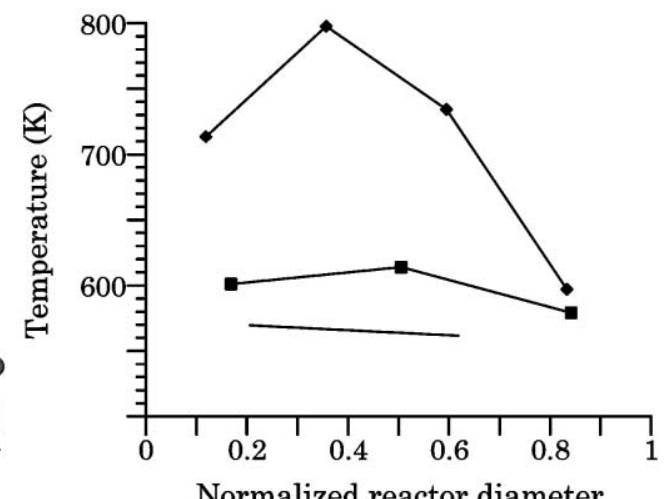

Normalized reactor diameter

(b)

Fig. 9. Temperature profiles for different $\varphi$ values: (a) longitudinal profiles in the reactor centre; (b) radial profiles in the hot-spots $\left(z^{*}\right)$.

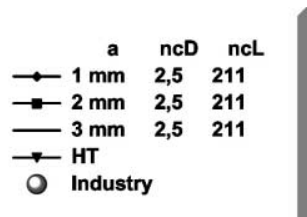

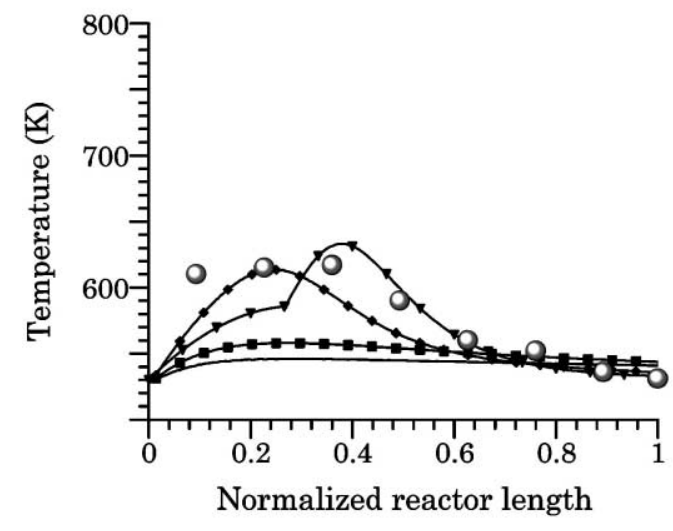

(a)

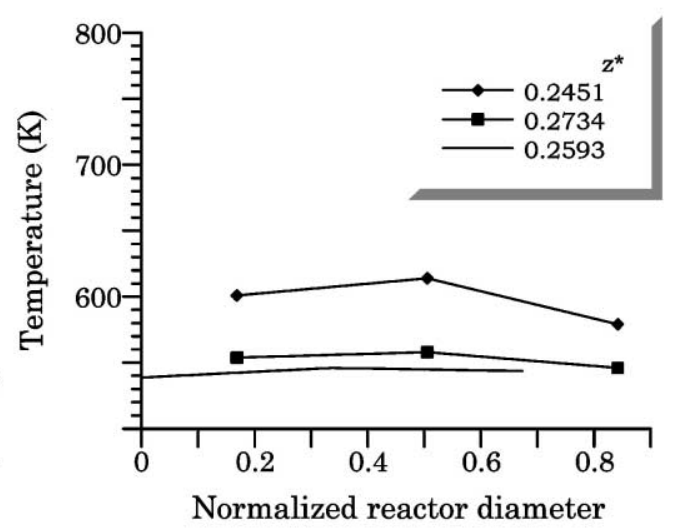

(b)

Fig. 10. Temperature profiles for different $a$ values: (a) longitudinal profiles in the reactor centre; (b) radial profiles in the hot-spots $\left(z^{*}\right)$. 


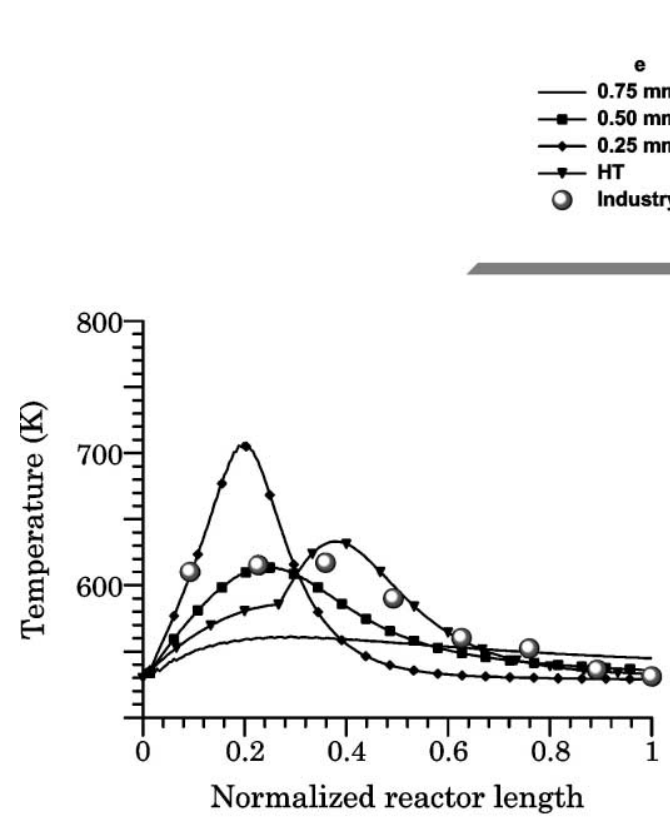

(a)

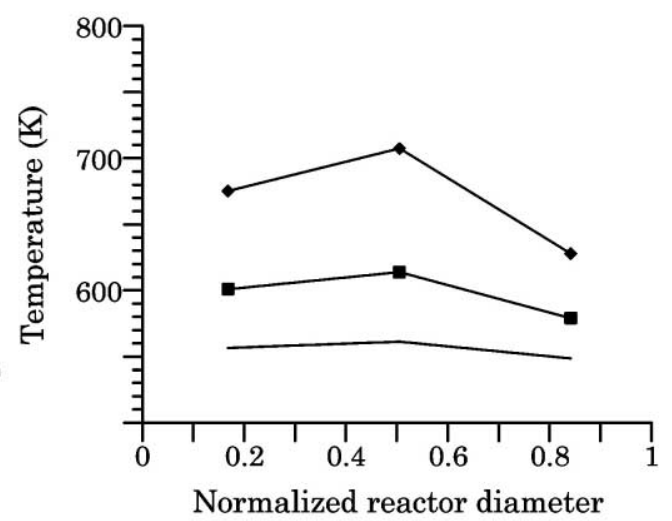

(b)

Fig. 11. Temperature profiles for different $e$ values: (a) longitudinal profiles in the reactor centre; (b) radial profiles in the hot-spots $\left(z^{*}\right)$.

predictions for the pressure drop is less than $10 \%$ and that the increase of the number of cells (lower $\lambda$ 's) also increases the pressure drop. Figs. 9-11 show the effect of the others parameters of the corrugated structure in the axial temperature profiles (in a) and in the radial gradients corresponding to the hot-spots (in b). Concerning the effect of the crossflow angle, $\varphi$, Fig. 9a and $\mathrm{b}$ shows the axial and radial temperature profiles for different values $\left(\varphi=30^{\circ}: \mathrm{ncD}=\frac{11}{2}, \mathrm{ncL}=298\right.$; $\varphi=45^{\circ}: \mathrm{ncD}=\frac{21}{2}, \mathrm{ncL}=211 ; \varphi=60^{\circ}: \mathrm{ncD}=\frac{31}{2}$, $\mathrm{ncL}=172$ ). The decrease of $\varphi$ will decrease the total number of cells inside the reactor and therefore the hot-spots will be reduced as well as the radial gradients. The temperature profiles obtained with different values of the height of the corrugation and represented in Fig. 10a and $\mathrm{b}\left(a=1,2\right.$, and $3 \mathrm{~mm}: \mathrm{ncD}=\frac{21}{2}$, $\mathrm{ncL}=211$ ) show that the temperature decreases when $a$ increases, due to the lower quantity of catalyst inside the reactor. Moreover, in Fig. 11a and b, one can observe the effect of the thickness of the corrugation $\left(e=0.75,0.5\right.$, and $0.25 \mathrm{~mm}: \mathrm{ncD}=\frac{21}{2}, \mathrm{ncL}=$ 211 ), being possible to conclude that the decrease of $e$ leads also to less catalyst and consequentially lower hot-spots are obtained.

\section{Conclusions}

The behaviour of a fixed-bed reactor where the partial oxidation of methanol to formaldehyde takes place is analysed when a structured packing is used. The simulations made to test the various parameters of the structure contributed to a better understanding of the behaviour of this type of packing and one could conclude that it is possible to optimise the structure in order to obtain the desired objectives by altering the parameters of the corrugated structure. Different amplitudes of the corrugation, $\lambda$, will lead to different dimensions of the cell, which, in turn, define the number of cells contained in the reactor. Higher values of $\lambda$ imply a low number of larger cells with less catalyst available and, consequently, less reaction rates and lower temperatures. For lower values of $\lambda$, the volume of the cell is lower and therefore more 
cells fit into the reactor. With more catalyst available, the reaction increases, raising the temperature. If the cell size is too small, it exists the possibility of a run away of the temperature. Similar analysis can be made in respect to the influence of the other parameters. In what concerns the crossflow angle, one can conclude that the lower is this angle the higher is the capacity for fluid mixing, leading then to reduced temperatures. The height and the thickness of the corrugations can also affect the behaviour of the system being then necessary to select the appropriate values, which will correspond to an adequate compromise of the hot-spots and the yield of the process. The reduction of the temperature is due to the high capacity of homogenisation of the structure, increasing the radial heat and mass transfer. The structure forces the fluid to flow diagonally placing it in contact with the reactor wall where heat transfer occurs. This enhancement is of extreme importance in the decrease of the catalyst deactivation and consequent diminishing of operation costs. The catalyst structure can also perform with a lower pressure drop by reducing the friction in the fluid flow typical of fixed beds. The fluid flows more freely between the corrugations due to a higher passage area.
Therefore, some general conclusions concerning the catalyst and its structure can be made as a result of this study, which mainly point out the possibility of reducing the overall temperature without diminishing the reaction yield. Moreover, the crosswise corrugated structures will lead to lower pressure drops along the bed also favouring energy savings.

\section{References}

[1] J.C. Godfrey, Static mixer, in: N. Harnbey, A.W. Nienow (Eds.), Mixing in the Process Industries, Butterworth Series in Chemical Engineering, London, 1989 (Chapter 13).

[2] J.P. Stringaro, J. Luder, Chem. Plants Process. 7 (1992).

[3] G. Gaiser, Heat Transfer 51 (1994) 261-266.

[4] G. Gaiser, Inst. Chem. Eng. Symp. Ser. 136 (1994) 431-440.

[5] M.H. Pahl, E. Muschelknautz, Int. Chem. Eng. 22 (1982) 197.

[6] F. Stoter, Z. Olujic, J. de Graauw, Chem. Eng. J. 53 (1993) $55-66$.

[7] R.B. Bird, W.E. Stewart, E.N. Lightfoot, Transport Phenomena, Wiley, New York, 1960.

[8] M. Dente, A. Collina, I. Pasquon, Chim. Ind. 48 (1966) 581588.

[9] M. Dente, A. Collina, Chim. Ind. 47 (1965) 821-829.

[10] R.B. Bird, Chem. Eng. Sci. 6 (1957) 128-181.

[11] M.J. Quina, R.M. Quinta-Ferreira, Chem. Eng. J. 75 (1999) 149-159. 